\title{
Microstar cavities: An alternative concept for the confinement of light
}

\author{
Julius Kullig, ${ }^{1, *}$ Xuefeng Jiang, ${ }^{2}$ Lan Yang $\odot,{ }^{2}$ and Jan Wiersig $\oplus^{1, \dagger}$ \\ ${ }^{1}$ Institut für Physik, Otto-von-Guericke-Universität Magdeburg, Postfach 4120, D-39016 Magdeburg, Germany \\ ${ }^{2}$ Department of Electrical and Systems Engineering, Washington University in St. Louis, St. Louis, Missouri 63130, USA
}

(Received 22 November 2019; accepted 5 March 2020; published 19 March 2020)

\begin{abstract}
In this Rapid Communication we report on an alternative concept to confine light in a microcavity. The traditional approaches are either based on total internal reflection at a dielectric interface or utilize a photonic band gap in a periodic structure such as Bragg mirrors or photonic crystals. In contrast, the concept presented here completely avoids these mechanisms. The light confinement is rather based on rays that are sequentially transmitted perfectly at the cavity's interface using Brewster's angle. These rays leave and reenter the cavity on closed periodic orbits without loss of intensity. Accordingly, in wave optics, high-quality modes with fascinating properties arise.
\end{abstract}

DOI: 10.1103/PhysRevResearch.2.012072

In 1878 Lord Rayleigh performed a pioneering experiment in St. Paul's Cathedral where a whisper could be heard around the gallery but not in the dome's center [1]. The explanation for this phenomenon is based on sound waves traveling almost lossless along the gallery due to successive reflections at the wall. Hence, the sound waves are confined inside the dome. For the confinement of light on the microscale two mechanisms are known so far which are either based on a photonic band gap [2] or on sequential total internal reflections at an dielectric interface $[3,4]$. Microcavities based on the latter phenomenon are also called whisperinggallery (WG) cavities due to their similarity to the sound wave experiments [see Fig. 1(a)]. In terms of wave optics WG cavities exhibit long-lived modes [see Fig. 1(b)] with a (slightly damped) time-harmonic behavior as solutions of Maxwell's equations. Nowadays, several types of WG cavities have been developed, e.g., microdisks [5-8], microspheres [9], or microtoroids $[10,11]$. Intentionally perturbing such WG cavities is used for many important applications such as microlasers with directional [12-20] and speckle-free [21] emission, sensors [22-25], gyroscopes [26], orbital angular momentum lasers [27], or in telecommunication technology [28]. Moreover, microcavities are excellent model systems for fundamental research in non-Hermitian physics of open systems [29] and quantum chaos [13,30,31]. However, in contrast to the sound waves used in Lord Rayleigh's experiments, light has additional unique properties. One of them is the perfect transmission at Brewster's angle if the light is polarized such that the magnetic field is perpendicular to the microdisk's plane [transverse-electric (TE) polarization].

\footnotetext{
*julius.kullig@ovgu.de

†jan.wiersig@ovgu.de

Published by the American Physical Society under the terms of the Creative Commons Attribution 4.0 International license. Further distribution of this work must maintain attribution to the author(s) and the published article's title, journal citation, and DOI.
}

In this Rapid Communication we demonstrate that perfect transmission at Brewster's angle leads to another concept to confine light. In contrast to macroscopic optical devices using Brewster's angle [32], our concept avoids total internal reflection and allows for a cavity design on the microscale. As shown in Fig. 1(c), a properly designed star-shaped cavity guides rays along closed orbits via sequential transmissions at Brewster's angle. Hence, these rays do not underlie losses from beam splitting or radiation into free space. We show that in wave optics long-lived modes arise that are supported by these trapped rays [see Fig. 1(d)]. The long-lived modes, which we call Brewster modes, have some fascinating properties. For example, a considerable fraction of the mode distribution is outside of the cavity, which may hold great potential for enhanced light-matter interaction, such as sensing, imaging, and trapping [33,34]. Moreover, we show that the Brewster modes are the only long-lived modes in the microstar cavity. That might be advantageous for, e.g., single-mode lasing and mode selection [35-37]. Via a slight convex deformation, the $Q$ factor of the Brewster modes can be improved further due to the stabilization of the ray dynamics around the periodic orbit.

First, the star-shaped cavity is designed by exploiting the ray dynamics. For this purpose, we consider a quasi-twodimensional cavity with a homogeneous effective refractive index $n$. Outside of the cavity the refractive index is assumed to be unity. Furthermore, we restrict ourselves to TE polarization, where at the cavity's dielectric interface at Brewster's angle $\chi_{\mathrm{B}}=\arctan (1 / n)$ a ray is fully transmitted. Hence, in order to construct the star shape with periodic ray trajectories that do not lose intensity over time, we need to fulfill the following conditions. (i) All of the ray's outgoing transmissions should occur with an angle of incidence $\chi=\chi_{\mathrm{B}}$. (ii) For a microstar with $v=3,4,5, \ldots$ spikes, it is required that at each spike the outgoing and the incoming transmissions change the ray's propagation direction by $2 \pi / v$ via diffraction. Both conditions (i) and (ii) are achieved by selecting the opening angle $\alpha$ of the spikes according to Brewster's angle as $\alpha=2 \chi_{\mathrm{B}}$ [see Fig. 2(a)], 


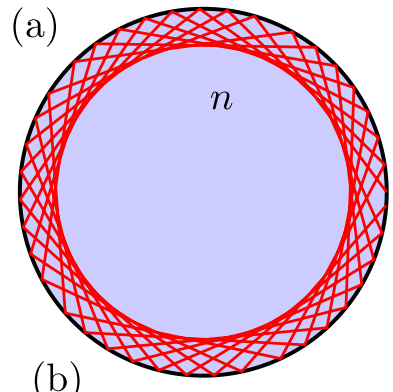

(b)
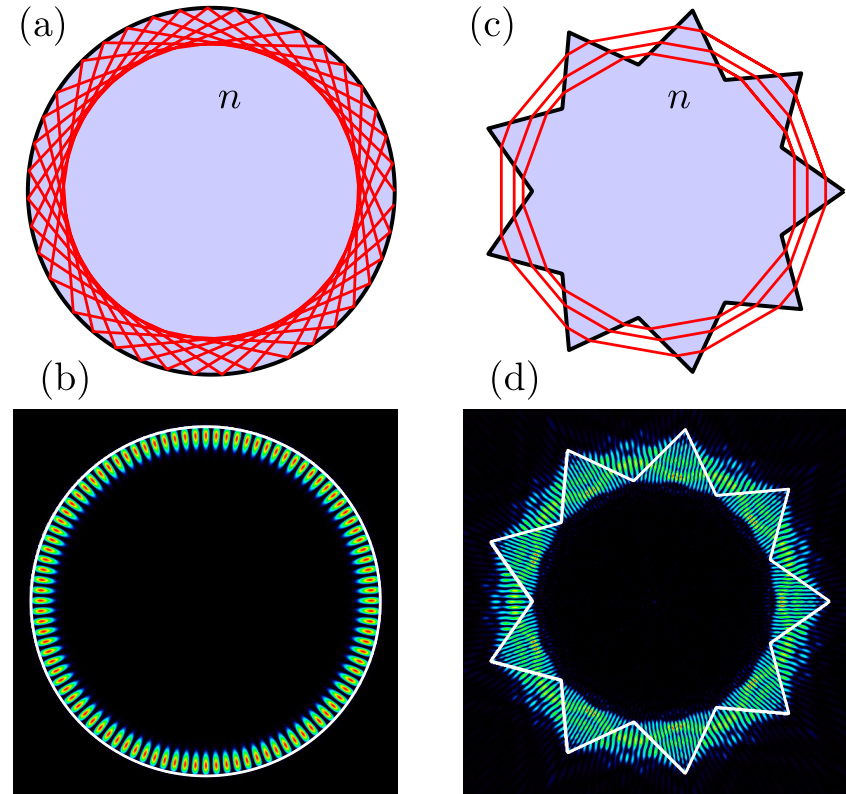

(d)

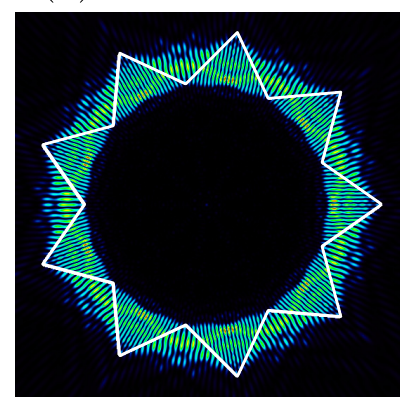

FIG. 1. The ray dynamics (a) in a whispering-gallery cavity and (c) in a microstar cavity with nine spikes is shown by red solid curves. (b) and (d) show the corresponding intensity patterns of a typical long-lived mode.

and simultaneously selecting the refractive index of the cavity as

$$
n=\frac{\sin (\pi / \nu)+1}{\cos (\pi / \nu)} .
$$

For the microstar shown in Fig. 1(c), Eq. (1) gives $n=1.4281$ for $v=9$.

To analyze the ray dynamics in more detail, a Poincaré section $\Sigma$ [38] is introduced at $y=0$ and $x>0$ [see Fig. 2(a)]. The conjugate momentum is thus given by $\sin \theta$, where $\theta$ is the angle of a ray and the ordinate when passing the section $\Sigma$. As it can be seen in Fig. 2(b), the microstar possesses a family of marginally stable and lossless periodic orbits at $\sin \theta=0$. However, rays with a finite $\sin \theta$ rapidly radiate out of the system after a few transmissions. In order to stabilize the dynamics we introduce a slight (convex) deformation of each line segment of the microstar. The idea behind it is to focus the rays with a slight but finite tangential momentum component $\sin \theta$ around a central periodic orbit. The deformation is defined for the first line segment with a polar angle $\phi \in[0, \pi / \nu]$ by the radius function $r_{\mathrm{seg}}(\phi)$ as

$$
\frac{r_{\mathrm{seg}}(\phi)}{R}=\frac{1}{n \sin \phi+\cos \phi}-\epsilon\left(\phi-\phi_{0}\right)^{2},
$$

where $\epsilon$ is a deformation parameter, $\phi_{0}=\pi /(2 v)$ is the polar angle of the central periodic orbit's first transmission, and $R$ is a typical scale for the radius of the microstar [see Fig. 2(a)]. For $\epsilon=0$, Eq. (2) describes a microstar with straight-line segments, whereas for $\epsilon>0(\epsilon<0)$ all line segments are deformed accordingly such that the deformation has a focusing (defocusing) effect. In the case of a focusing deformation the central periodic orbit at $[x, \sin \theta]=\left[r_{\mathrm{seg}}\left(\phi_{0}\right) \cos \phi_{0}, 0\right]$ becomes a stable elliptical fixed point surrounded by a region of regular motion in the phase space [see Figs. 2(c)-2(e)].
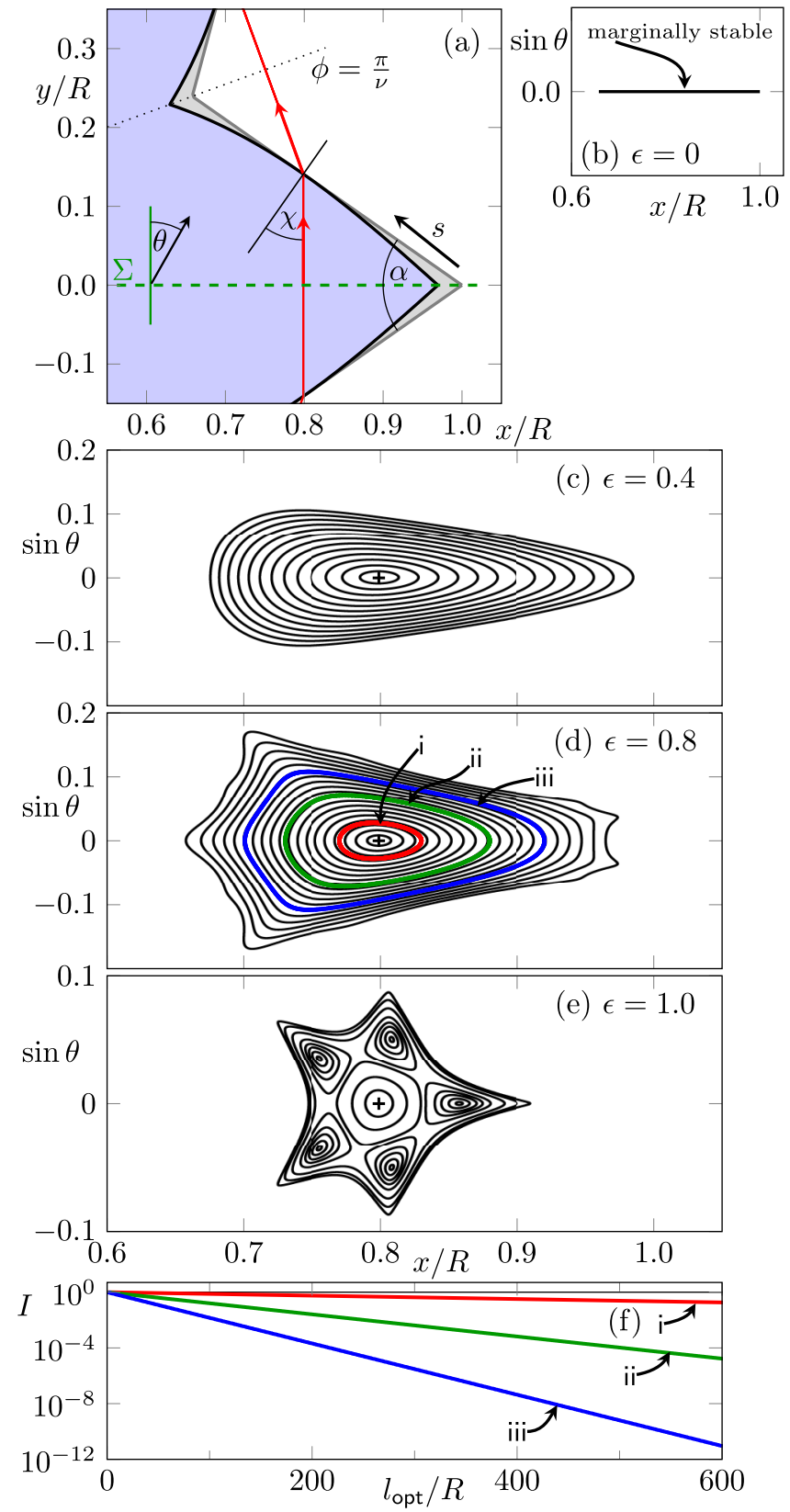

FIG. 2. (a) A magnification of the ray dynamics in a microstar cavity with $v=9$ spikes. The microstar with straight-line segments $(\epsilon=0)$ is shown as a light gray solid curve. The deformed microstar with $\epsilon=1$ is illustrated by a thick solid black curve. The phase-space section $\Sigma$ is shown as a dashed green curve. A phase-space portrait of (b) a straight-lined microstar and (c)-(e) a deformed microstar is shown by solid curves. The central periodic orbit is marked by a plus. The intensity loss of the orbits represented by thick (labeled) curves in (d) is plotted in (f) vs their optical path length. The thin curve in (f) is the constant intensity of the central periodic orbit.

Since all of the periodic orbit's transmissions are still at Brewster's angle, it does not suffer losses over time. For larger deformations additional Poincaré-Birkhoff resonance chains [38] are formed [cf. Fig. 2(e)]. However, orbits in the regular region around the central periodic orbit are also trapped in the deformed microstar for arbitrary long times but they are 
not transmitted exactly at Brewster's angle. Therefore, these orbits lose a slight fraction of intensity in each transmission as shown in Fig. 2(f).

Next, the wave optics in the microstar cavity is investigated. Therefore, we solve Maxwell's equations in the effective index approximation for quasi-two-dimensional microdisk cavities [39]. This standard approach results in a scalar mode equation

$$
\left[\nabla^{2}+n(\vec{r})^{2} \frac{\omega^{2}}{c^{2}}\right] \psi(\vec{r})=0
$$

for the wave function $\psi$. In the case of TE polarization $\psi$ represents the $z$ component of the magnetic field as $H_{z}=$ $\operatorname{Re}\left(\psi e^{-i \omega t}\right) ; c$ is the speed of light in vacuum. It is common to rescale the complex frequency $\omega$ to the dimensionless quantity $k R=\omega R / c$. The boundary conditions at the dielectric interface for TE polarization are the continuity of $\psi$ and its scaled normal derivative $n^{-2} \partial_{\vec{v}} \psi$. We solve the mode equation for the microstar numerically with the boundary element method (BEM) [40]. While doing so the sharp corners are slightly rounded by a parabolic interpolation. As a first example, a microstar with $v=9$ spikes and straight-line segments $(\epsilon=0)$ is considered. A section of the complex frequency plane is shown in Fig. 3(a). Note that the long-lived modes, i.e., the modes that are close to the real frequency axis, correspond to Brewster modes. The transmission at Brewster's angle can be seen more directly in the Husimi function [41] [see Fig. 3(e)] which is a phase-space representation of a mode. Here, the phase space is spanned by the arclength $s$ along the cavity's interface and the angle of incidence $\chi$ [see Fig. 2(a)]. Note that the mode is clearly localized at Brewster's angle and below the critical line of total internal reflection. The localization of the mode at Brewster's angle becomes even more obvious when the Husimi function is integrated over the arclength $s$ of the cavity which reveals an angle-ofincidence distribution $\mathcal{W}(\sin \chi)$. As shown in Fig. 3(f), this distribution is centered around Brewster's angle. Furthermore, note that other modes which are not related to Brewster's angle are typically deep in the complex frequency plane and therefore have a small $Q$ factor, $Q=-\operatorname{Re} k R /(2 \operatorname{Im} k R)$. It is remarkable that the Brewster modes are almost equally spaced in Re $k R$ [see Fig. 3(a)]. The spacing $\Delta k R$ can be estimated by setting the optical path length $l_{\text {opt }}=\int n(\vec{r}) d \vec{r}$ of the periodic orbit to a multiple of the (local) wavelength $\lambda=2 \pi / k$. The spacing between two of such quantizing wavelengths is given by

$$
\Delta k R=\frac{\pi / v}{\sin (\pi / \nu)},
$$

which is related to a free spectral range $\Delta \lambda_{\mathrm{FSR}}=$ $\lambda^{2} \Delta k R /(2 \pi R)$. Note that at each of these quantizing frequencies two Brewster modes exists: one with even and one with odd parity with respect to a mirror-reflection symmetry line of the cavity. The frequency spacing between such a pair of modes is rather small and cannot be seen in Fig. 3(a) but can be seen in the magnification in Fig. 3(d).

The $Q$ factor of the Brewster modes increases with a higher real part of the complex frequency. In order to roughly estimate the $Q$ factor of the Brewster modes we consider a Gaussian beam along the central periodic orbit that is
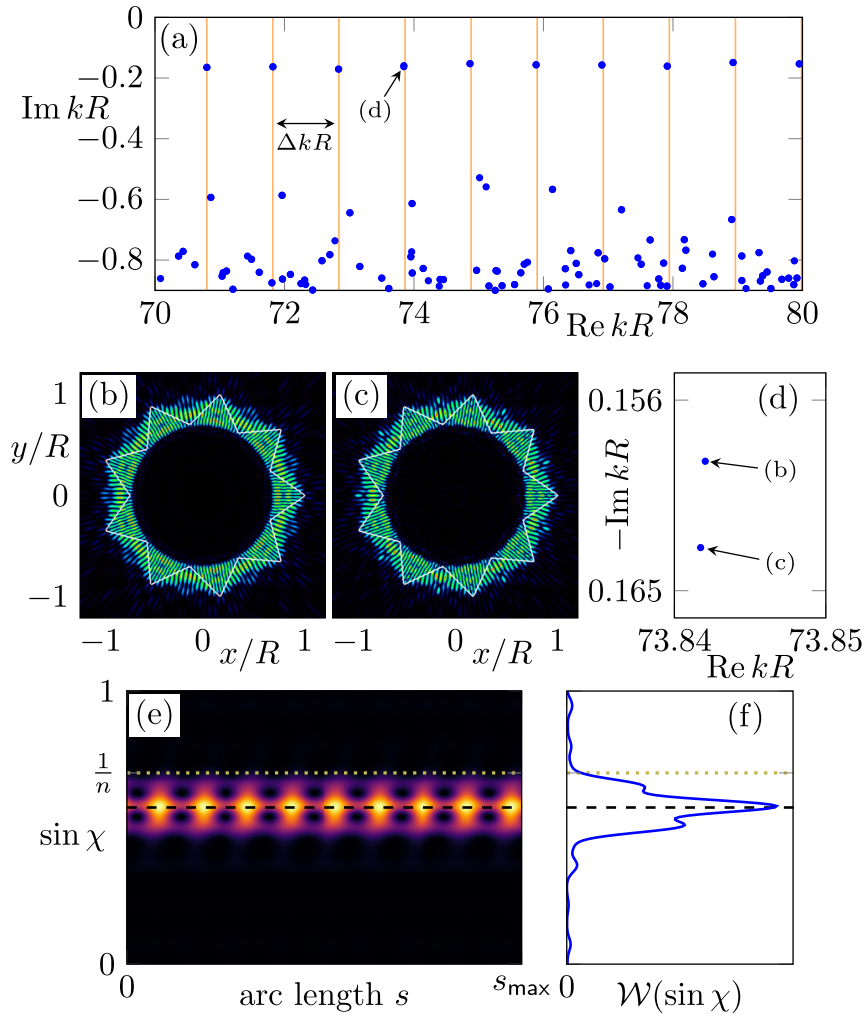

FIG. 3. (a) Complex dimensionless frequency plane for a ninespiked microstar. Optical modes are shown as blue dots. The spacing between quantizing frequencies given by Eq. (4) is illustrated by orange lines. In (b) [(c)] the intensity pattern of the odd [even] parity mode at $k R \approx 73.84-i 0.16$ [see magnification of the complex frequency space in (d)] is shown. (e) The Husimi function of the mode shown in (b). (f) The angle-of-incidence distribution $\mathcal{W}(\sin \chi)$ for the same mode is shown as a solid blue curve. In (e) and (f) the critical line of total internal reflection (Brewster's angle) is indicated by a dotted yellow (dashed black) line.

transmitted at $\phi=\pi /(2 v)$ [see Fig. 2(a)]. The Gaussian beam is described by an angle-of-incidence distribution

$$
f(p)=\exp \left[-a_{v}^{2} \operatorname{Re}(n k R)\left(p-p_{\mathrm{B}}\right)^{2} / 4\right],
$$

where $p_{(\mathrm{B})}=\sin \chi_{(\mathrm{B})}$ and $a_{v}$ is a (fitted) $k R$ independent width parameter which depends on the number of spikes $v$. The fraction of intensity which is not transmitted but reflected provokes losses. Only the intensity fraction $\mu_{k}$ which is transmitted at the interface can proceed on the periodic orbit. Using the Fresnel intensity reflection coefficient $R(p)$ we can estimate $\mu_{k}=\int_{0}^{1}[1-R(p)] f^{2}(p) d p / \int_{0}^{1} f^{2}(p) d p$. Next, we exploit that the optical path length $l_{\text {opt }}$ is equal for all (marginally stable) periodic orbits. Thus, the time $\tau$ for the propagation from $\phi=0$ to $\phi=\pi / \nu$ can be approximated from the orbit at the tip of the spike as $\tau=\sin (\pi / v) R / c \approx$ $\pi R /(v c)$. Within this time $\tau$ a mode loses intensity according to $|\psi(\vec{r}, t+\tau)|^{2}=\exp (2 \operatorname{Im} \omega \tau)|\psi(\vec{r}, t)|^{2}$. Hence, comparing the factor $\exp (2 \operatorname{Im} \omega \tau)$ with $\mu_{k}$ yields

$$
Q=-\frac{\pi}{v} \frac{\operatorname{Re} k R}{\ln \mu_{k}}
$$



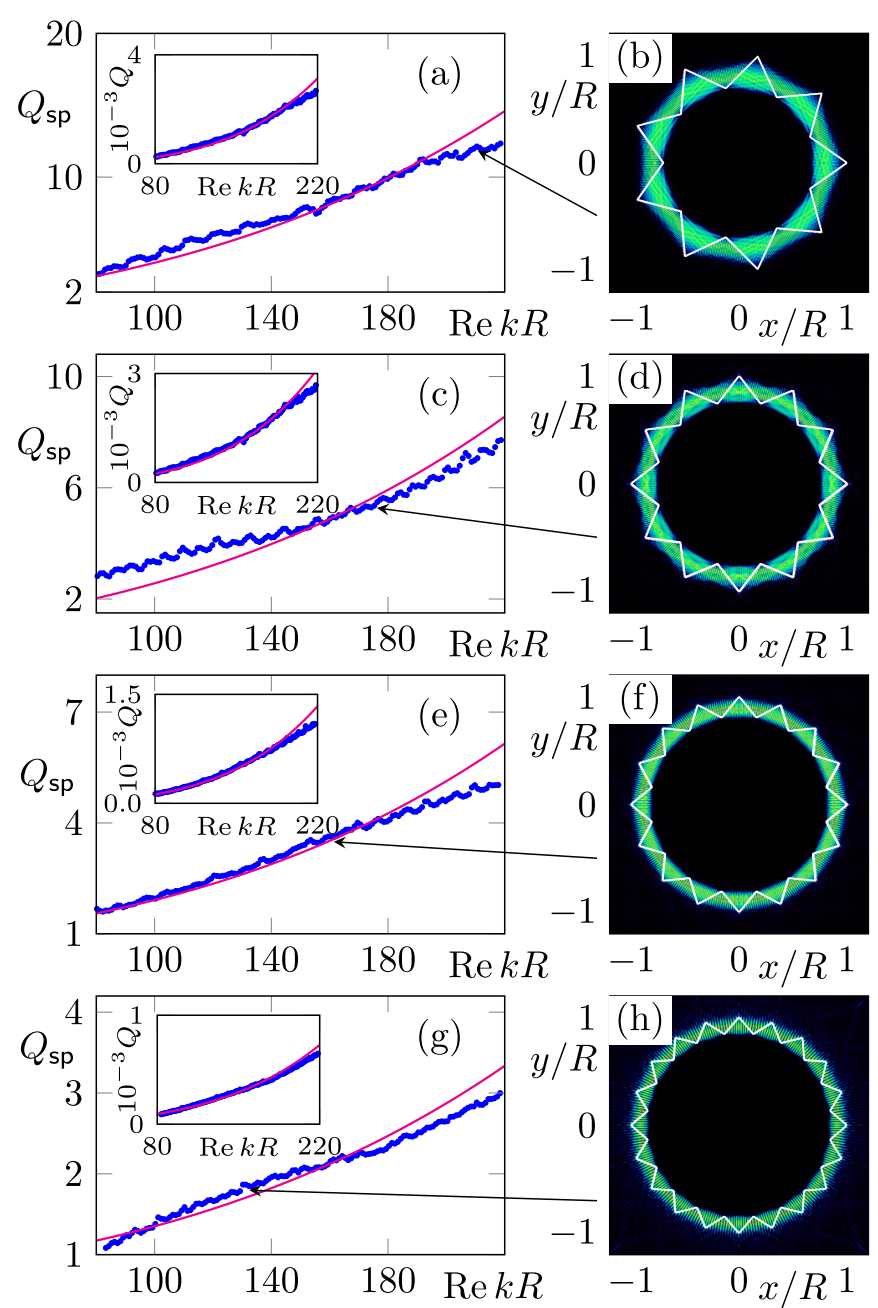

FIG. 4. The frequency-splitting quality $Q_{\mathrm{sp}}$ and in the insets the quality factor $Q$ of the Brewster modes for a microstar with (a) $v=$ 9 , (c) $v=12$, (e) $v=16$, and (g) $v=20$ spikes are shown as blue dots. The magenta solid curves are the estimates from Eqs. (6) and (7) with fitted $a_{9} \approx 1.0, a_{12} \approx 0.773, a_{16} \approx 0.662$, and $a_{20} \approx 0.543$. Examples of the mode's intensity patterns are shown on the right panel. The refractive indexes of the cavities are chosen according to Eq. (1).

as an estimate of the $Q$ factor. Another important quantity is the frequency-splitting quality $Q_{\mathrm{sp}}=-\operatorname{Re}\left(k_{j+1}-\right.$ $\left.k_{j}\right) / \operatorname{Im}\left(k_{j+1}+k_{j}\right)[42,43]$ between two sequential modes indicated by $j$ and $j+1$. For $Q_{\text {sp }} \gtrsim 2 / 3$ the modes can be distinguished as individual peaks in the spectrum. With Eq. (6) the frequency-splitting quality can be estimated as

$$
Q_{\mathrm{sp}}=-\frac{2 \pi}{v} \frac{\Delta k R}{\ln \mu_{k+\Delta k}+\ln \mu_{k}} .
$$

In Fig. 4 it is shown that these estimates for the $Q$ factor and the frequency-splitting quality nicely fit the numerical data obtained with BEM for different microstar cavities and over a large range of $\operatorname{Re} k R$. Note that a microstar with a smaller number of spikes tends to exhibit slightly larger $Q$ factors. This is consistent with the idea that a slight fraction of intensity is reflected at each dielectric interface [cf. Eq. (6)] which then causes losses.

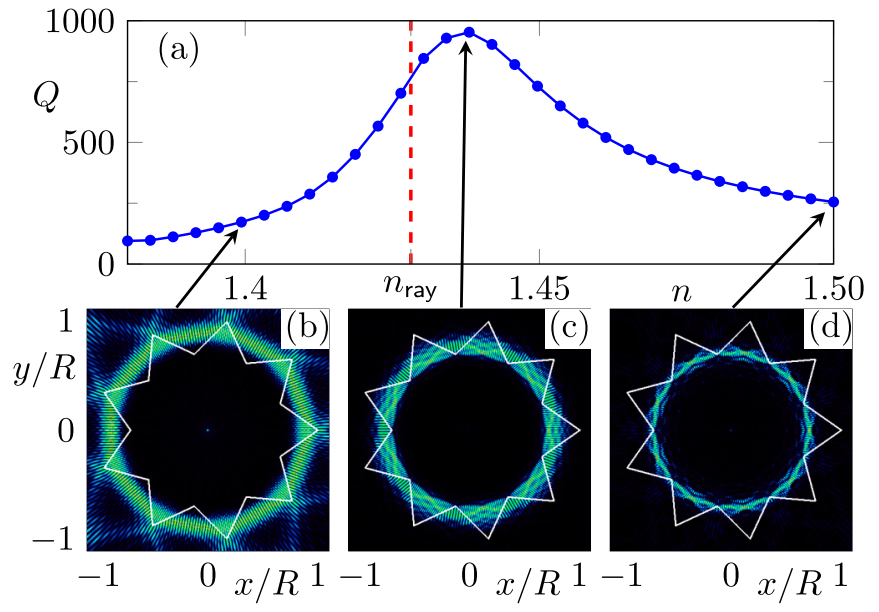

FIG. 5. (a) The (dots connected by solid curves) $Q$-factor dependency on the refractive index of the microstar is shown for the mode with $k R=128.83827-i 0.08396$ at $n=n_{\text {ray }}=1.42815$ (red dashed line) [see Eq. (1)]. The intensity mode patterns for $n=1.4$, $n=1.4375$, and $n=1.5$ are shown in (b)-(d).

In general, the $Q$ factor also depends on the refractive index $n$ of the cavity. For a WG cavity increasing the refractive index typically results in a larger $Q$ factor due to a decreasing of the evanescent leakage and scattering loss. However, for the microstar it is crucial that the refractive index is chosen correctly such that the transmissions along the periodic orbit are at Brewster's angle. This also manifests in the wave optics as the $Q$ factor has a peak at an optimal value of $n$ (see Fig. 5). Due to finite-wavelength corrections $[44,45]$

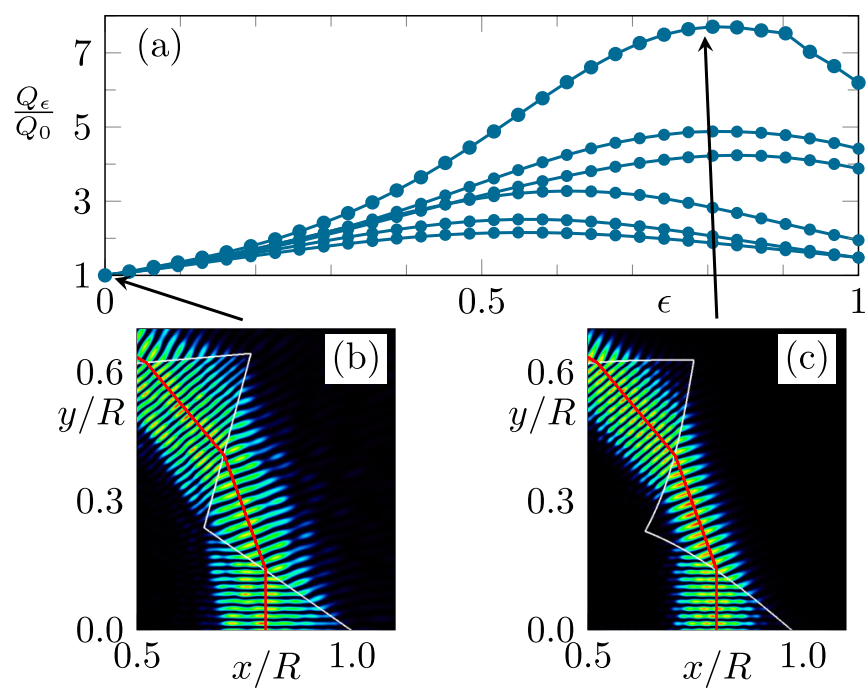

FIG. 6. The (dots connected by solid curves) enhancement of the $Q$ factor for some Brewster modes with $\operatorname{Re} k R \in[100,130]$ in a nine-spiked microstar cavity vs the deformation strength $\epsilon$ [see Eq. (2)]. In (b) [(c)] a magnification of the intensity pattern of the mode $k R=128.83827-i 0.08396[k R=129.40617-i 0.01107]$ for $\epsilon=0[\epsilon=0.8]$ is shown. The solid red curves represent the central periodic orbit. The refractive index is adjusted according to Eq. (1). 
the optimal refractive index is not exactly but close to the prediction from ray dynamics in Eq. (1). In experiments an adjustment of the refractive index can be realized by changing the environmental gas [34], the temperature [46], the pump power [47], or the cavity's material composition [48,49].

Next, in Fig. 6(a) it shown that a deformation of the microstar according to Eq. (2) can improve the $Q$ factor of the Brewster modes. Comparing the mode patterns for $\epsilon=0$ and $\epsilon=0.8$ reveals that the deformation has a focusing effect of the mode along the central periodic orbit. Note that in Fig. 6(a) the $Q$-factor enhancement is different for each mode. Usually, $\epsilon \in[0.5,1]$ is a good choice for all the investigated modes to achieve a significant improvement. The calculated $Q$ factors, e.g., $Q \approx 5845$ for the mode in Fig. 6(c), still might be at the lower end in comparison to WG cavities. At a similar refractive index $n$ and frequency Re $k R$ deformed WG cavities have $Q \sim 10^{3} \ldots 10^{4}[19,50]$. However, the modes in the microstar have the unique property that almost half of the mode's intensity $|\psi|^{2}$ is trapped outside of the cavity whereas
WG modes only leak evanescently to the surrounding area of the cavity.

In summary, we have demonstrated that a properly designed microstar cavity with an adjusted refractive index supports long-lived optical modes based on rays that are sequentially transmitted at Brewster's angle. The underlying mechanism is not based on total internal reflection nor on a photonic band gap. Therefore, the microstar cavity is fundamentally different from traditional cavities and promises additional applications. As optical modes have a finite wavelength they cannot localize precisely on a single periodic orbit and therefore undergo slight scattering losses leading to a finite quality factor. However, by slight deformations of the microstar's spikes the $Q$ factor of the Brewster modes can be improved further due to a stabilization of the ray dynamics around a central periodic orbit.

The authors acknowledge fruitful discussions with $\mathrm{M}$. Badel and C.-H. Yi.
[1] L. Rayleigh, The Theory of Sound (American edition: Dover, New York, 1945), first published 1877.

[2] E. Yablonovitch, Phys. Rev. Lett. 58, 2059 (1987).

[3] R. K. Chang and A. J. Campillo, Optical Processes in Microcavities, Advanced Series in Applied Physics Vol. 3 (World Scientific, Singapore, 1996).

[4] K. J. Vahala, Optical Microcavities, Advanced Series in Applied Physics Vol. 5 (World Scientific, Singapore, 2004).

[5] T. J. Kippenberg, J. Kalkman, A. Polman, and K. J. Vahala, Phys. Rev. A 74, 051802(R) (2006).

[6] S. L. McCall, A. F. J. Levi, R. E. Slusher, S. J. Pearton, and R. A. Logan, Appl. Phys. Lett. 60, 289 (1992).

[7] C. P. Michael, K. Srinivasan, T. J. Johnson, O. Painter, K. H. Lee, K. Hennessy, H. Kim, and E. Hu, Appl. Phys. Lett. 90, 051108 (2007).

[8] A. C. Tamboli, E. D. Haberer, R. Sharma, K. W. Lee, S. Nakamura, and E. L. Hu, Nat. Photonics 1, 61 (2007).

[9] L. Collot, V. Lefevre-Seguin, M. Brune, J. Raimond, and S. Haroche, Europhys. Lett. 23, 327 (1993).

[10] V. S. Ilchenko, M. L. Gorodetsky, X. S. Yao, and L. Maleki, Opt. Lett. 26, 256 (2001).

[11] D. K. Armani, T. J. Kippenberg, S. M. Spillane, and K. J. Vahala, Nature (London) 421, 925 (2003).

[12] J. U. Nöckel, A. D. Stone, G. Chen, H. L. Grossman, and R. K. Chang, Opt. Lett. 21, 1609 (1996).

[13] J. U. Nöckel and A. D. Stone, Nature (London) 385, 45 (1997).

[14] J. Wiersig and M. Hentschel, Phys. Rev. Lett. 100, 033901 (2008).

[15] Q. J. Wang, C. Yan, N. Yu, J. Unterhinninghofen, J. Wiersig, C. Pflügl, L. Diehl, T. Edamura, M. Yamanishi, H. Kan et al., Proc. Natl. Acad. Sci. USA 107, 22407 (2010).

[16] S. Shinohara, M. Hentschel, J. Wiersig, T. Sasaki, and T. Harayama, Phys. Rev. A 80, 031801(R) (2009).

[17] F. Albert, C. Hopfmann, A. Eberspächer, F. Arnold, M. Emmerling, C. Schneider, S. Höfling, A. Forchel, M. Kamp, J. Wiersig et al., Appl. Phys. Lett. 101, 021116 (2012).
[18] Q. J. Wang, C. Yan, L. Diehl, M. Hentschel, J. Wiersig, N. Yu, C. Pflügl, M. A. Belkin, T. Edamura, M. Yamanishi et al., New J. Phys. 11, 125018 (2009).

[19] M. Schermer, S. Bittner, G. Singh, C. Ulysee, M. Lebental, and J. Wiersig, Appl. Phys. Lett. 106, 101107 (2015).

[20] X.-F. Jiang, Y.-F. Xiao, C.-L. Zou, L. He, C.-H. Dong, B.-B. Li, Y. Li, F.-W. Sun, L. Yang, and Q. Gong, Adv. Mater. 24, 260 (2012).

[21] B. Redding, A. Cerjan, X. Huang, M. L. Lee, A. D. Stone, M. A. Choma, and H. Cao, Proc. Natl. Acad. Sci. USA 112, 1304 (2015).

[22] F. Vollmer and S. Arnold, Nat. Methods 5, 591 (2008).

[23] A. M. Armani, R. P. Kulkarni, S. E. Fraser, R. C. Flagan, and K. J. Vahala, Science 317, 783 (2007).

[24] L. He, Ş. K. Özdemir, J. Zhu, W. Kim, and L. Yang, Nat. Nanotechnol. 6, 428 (2011).

[25] W. Chen, Ş. K. Özdemir, G. Zhao, J. Wiersig, and L. Yang, Nature (London) 548, 192 (2017).

[26] S. Sunada and T. Harayama, Opt. Express 15, 16245 (2007).

[27] P. Miao, Z. Zhang, J. Sun, W. Walasik, S. Longhi, N. M. Litchinitser, and L. Feng, Science 353, 464 (2016).

[28] T. Kippenberg, R. Holzwarth, and S. Diddams, Science 332, 555 (2011).

[29] H. Cao and J. Wiersig, Rev. Mod. Phys. 87, 61 (2015).

[30] S. Sunada, S. Shinohara, T. Fukushima, and T. Harayama, Phys. Rev. Lett. 116, 203903 (2016).

[31] X. Jiang, L. Shao, S.-X. Zhang, X. Yi, J. Wiersig, L. Wang, Q. Gong, M. Lončar, L. Yang, and Y.-F. Xiao, Science 358, 344 (2017).

[32] K. Lehmann, P. Johnston, and P. Rabinowitz, Appl. Opt. 48, 2966 (2009).

[33] H. A. Huckabay and R. C. Dunn, Sens. Actuators, B 160, 1262 (2011).

[34] F. Vollmer and L. Yang, Nanophotonics 1, 267 (2012).

[35] M. Fujita and T. Baba, IEEE J. Quantum Electron. 37, 1253 (2001).

[36] S. V. Boriskina, T. M. Benson, P. D. Sewell, and A. I. Nosich, IEEE J. Sel. Top. Quantum Electron. 12, 1175 (2006). 
[37] A. Schlehahn, F. Albert, C. Schneider, S. Höfling, S. Reitzenstein, J. Wiersig, and M. Kamp, Opt. Express 21, 15951 (2013).

[38] A. J. Lichtenberg and M. A. Lieberman, Regular and Chaotic Dynamics (Springer, Berlin, 1992).

[39] J. D. Jackson, Classical Electrodynamics (Wiley, New York, 1962).

[40] J. Wiersig, J. Opt. A: Pure Appl. Opt. 5, 53 (2002).

[41] M. Hentschel, H. Schomerus, and R. Schubert, Europhys. Lett. 62, 636 (2003).

[42] J. Wiersig, Phys. Rev. A 93, 033809 (2016).

[43] Ş. K. Özdemir, J. Zhu, L. He, and L. Yang, Phys. Rev. A 83, 033817 (2011).
[44] H. E. Tureci, H. G. L. Schwefel, A. D. Stone, and E. E. Narimanov, Opt. Express 10, 752 (2002).

[45] M. Hentschel and H. Schomerus, Phys. Rev. E 65, 045603(R) (2002).

[46] M. Benyoucef, J.-B. Shim, J. Wiersig, and O. G. Schmidt, Opt. Lett. 36, 1317 (2011).

[47] B. R. Bennett, R. A. Soref, and J. A. Del Alamo, IEEE J. Quantum Electron. 26, 113 (1990).

[48] D. Aspnes, S. Kelso, R. Logan, and R. Bhat, J. Appl. Phys. 60, 754 (1986).

[49] S. Adachi, J. Appl. Phys. 66, 6030 (1989).

[50] S. Liu, J. Wiersig, W. Sun, Y. Fan, L. Ge, J. Yang, S. Xiao, Q. Song, and H. Cao, Laser Photonics Rev. 12, 1800027 (2018). 